Supplement of

Gas-phase chemistry in the online multiscale NMMB/BSC Chemical Transport Model: Description and evaluation at global scale

Badia et al., 2016

Correspondence to: Oriol Jorba (oriol.jorba@ bsc.es)

Contents of this file:

Statistical Measures

Figures $\mathrm{S} 1$ to $\mathrm{S} 5$

Tables S1 to S3 


\section{Statistical Measures}

There are several metrics that are used by the modeling community to evaluate performances of AQMs (U.S.EPA, 1991; Cox and Tikvart, 1990; Russell and Dennis, 2000). The statistical indicators selected in this study are: Correlation coefficient (r: Eq. 11), Mean Bias (MB: Eq. 2) and Root Mean Square Error (RMSE: Eq 3).

$$
\begin{gathered}
r=\frac{1}{N} \frac{\sum_{i=1}^{N}\left(O_{i}-\bar{O}\right) \Delta\left(P_{i}-\bar{P}\right)}{\sigma_{O} \Delta \sigma_{P}} \\
M B=\frac{\sum_{i=1}^{N}\left(P_{i}-O_{i}\right)}{N} \\
R M S E=\sqrt{\frac{1}{N} \sum_{i=1}^{N}\left(P_{i}-O_{i}\right)^{2}}
\end{gathered}
$$

where $\sigma$ is the standard deviation and $P$ and $O$ denote the vector of model output and the vector observations, respectively. No threshold has been applied in the computation of the statistics. 


\section{Figures}
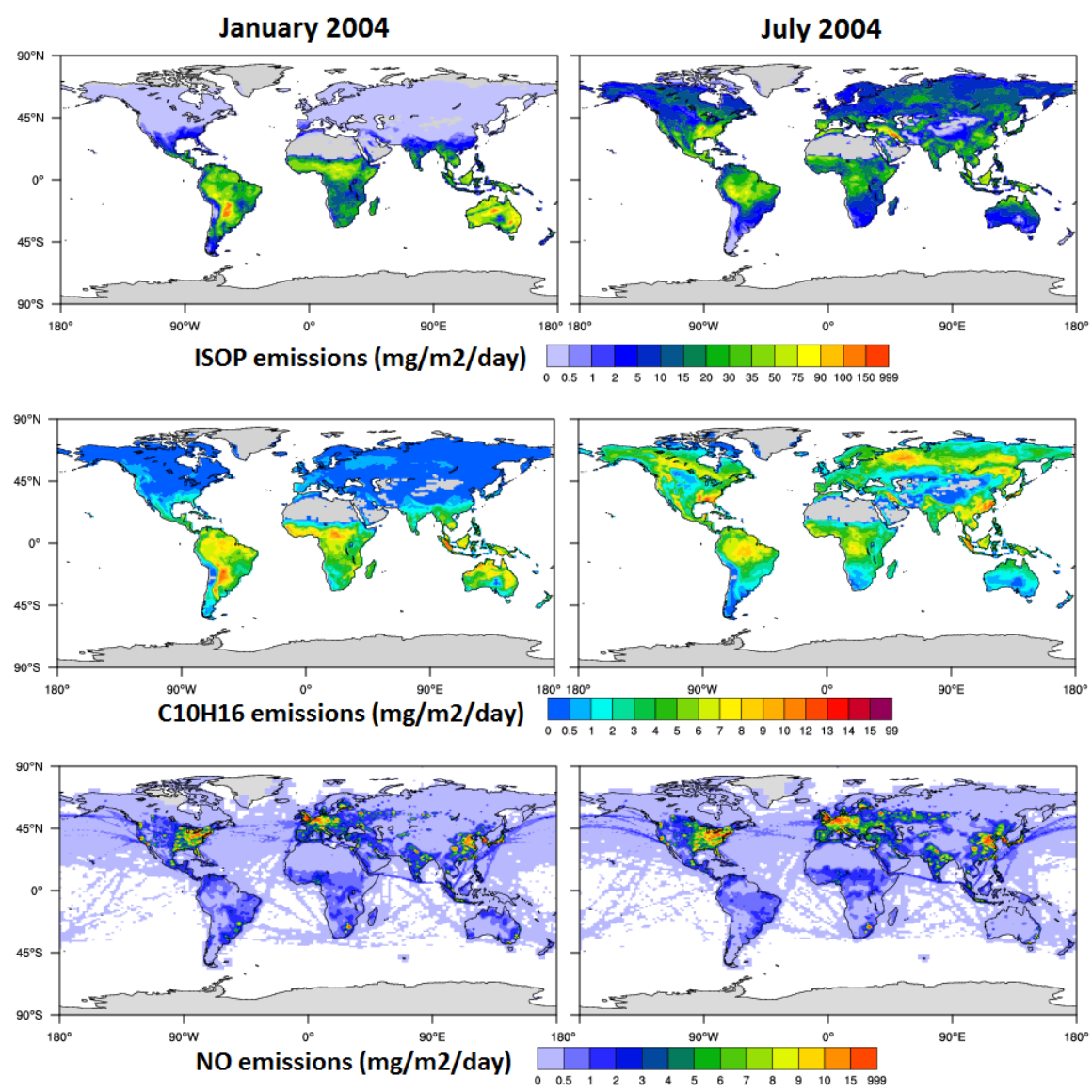

Figure S1: Biogenic emisions of isoprene (upper panel) and monoterpene (middle panel), from the on-line model MEGAN, and anthropogenic emissions of NO, from ACCMIP inventory, for January and July 2004 used in this model simulation 

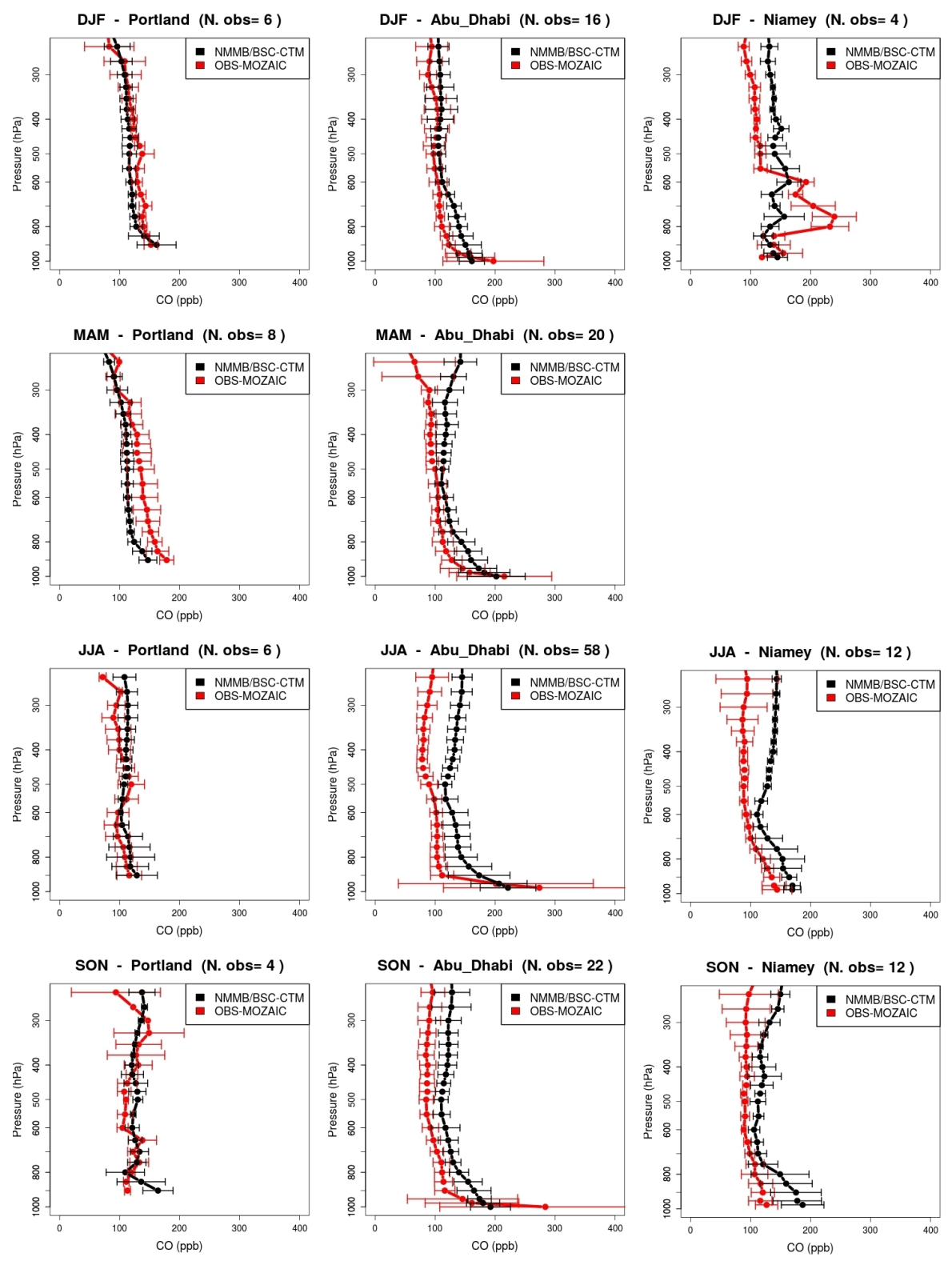

Figure S2: CO vertical profile seasonal averages over Portland, Abu Zabi and Niamey (from left to right) for the whole year 2004. Observations are in a solid red line and model data in a solid black line. The number of observations flights is given on the top of each plot. 

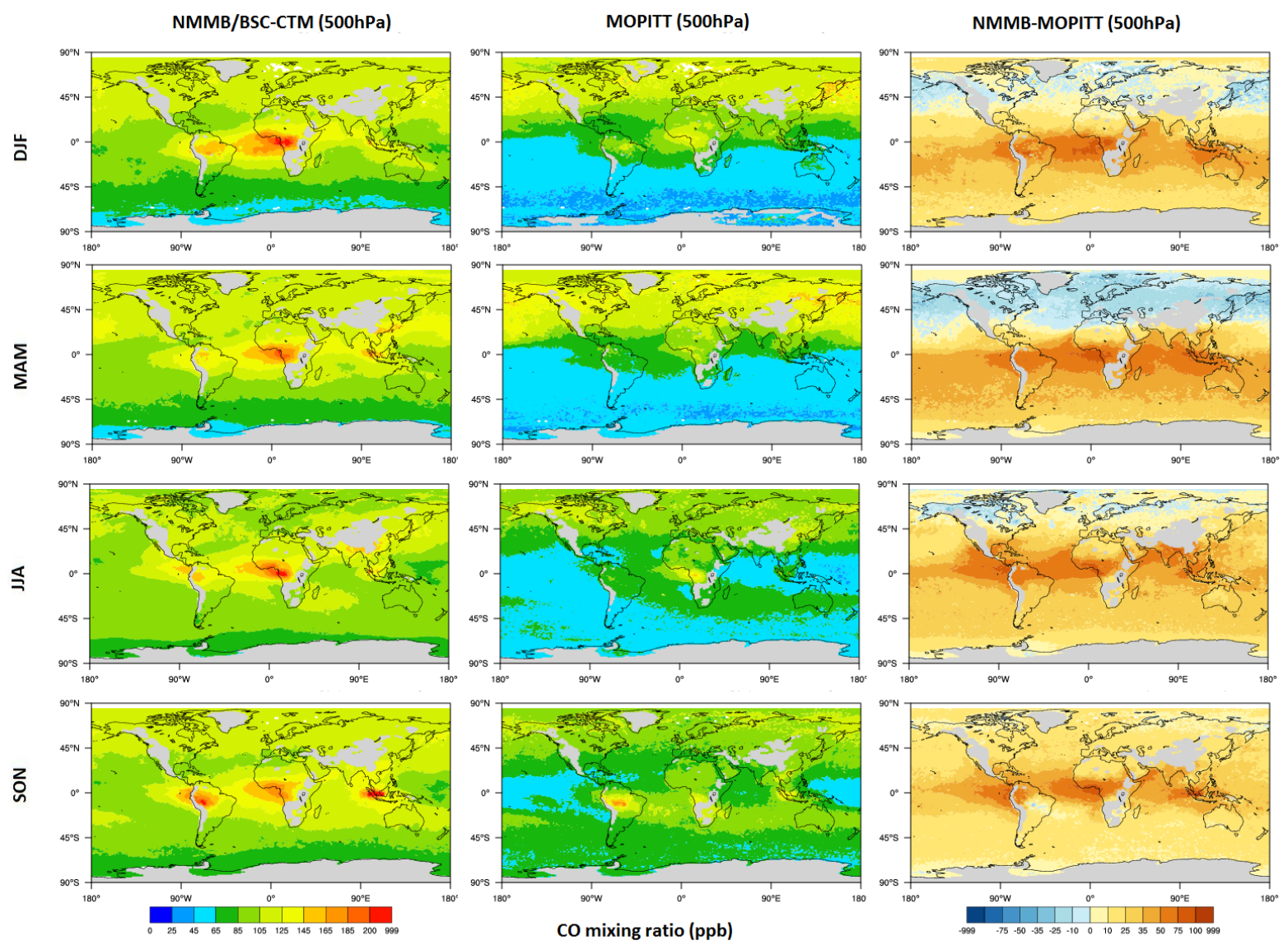

Figure S3: Comparison of modeled NMMB/BSC-CTM CO mixing ratio at 500hPa against satellite data (MOPITT) for (from top) DJF, MAM, JJA, and SON for the whole year 2004 in ppb. NMMB/BSC-CTM data is displayed in the left panel, MOPITT data in the middle panel and the bias in the right panel. 

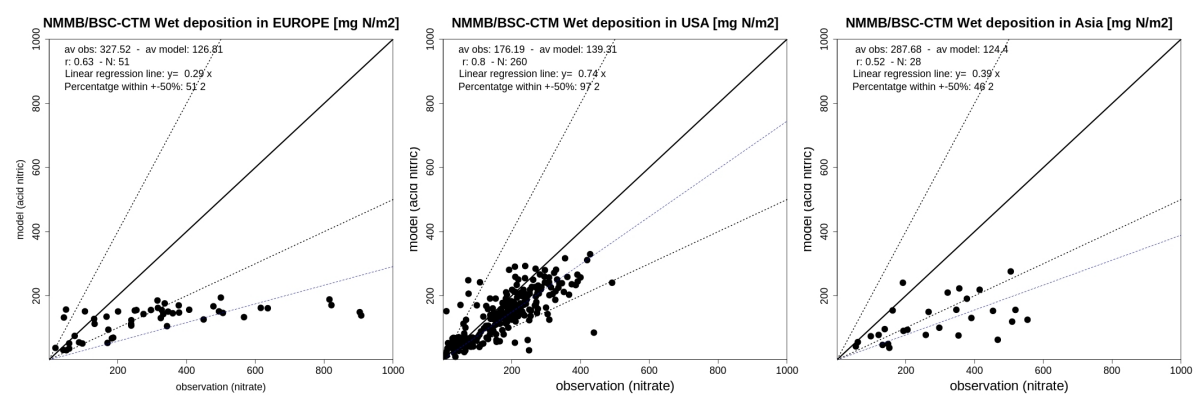

Figure S4: Scatter plots of the simulated $\mathrm{HNO}_{3}$ versus nitrate measurements for three networks: Europe (left panel), USA (middle panel) and Asia (right panel). Dashed lines have slopes equal to 2 and 0.5 . The dotted line is the result of the linear regression fitting through the origin. 

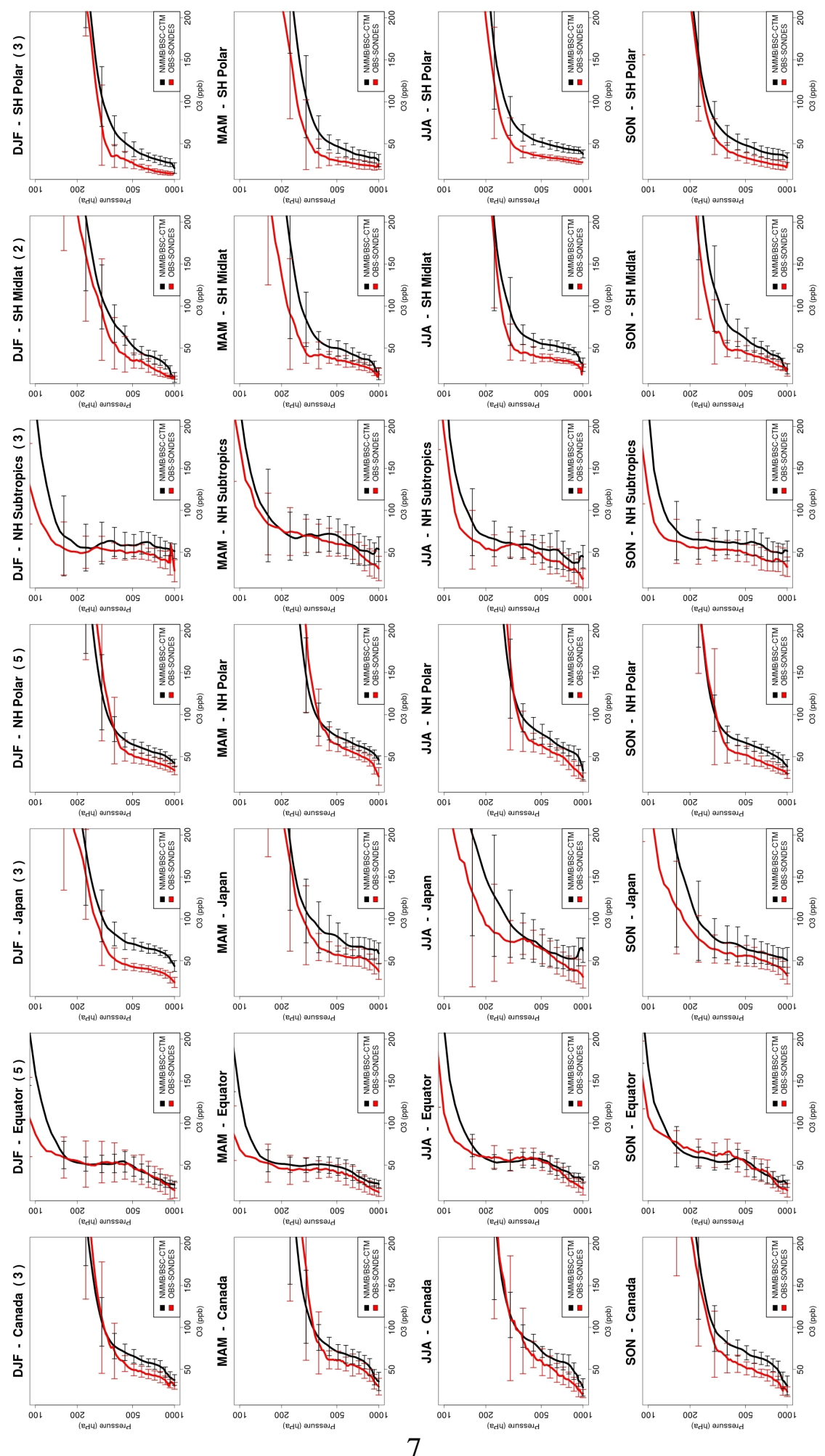

Figure S5: Comparison of ozonesonde measurements (red lines) and simulated (black lines) seasonal vertical profiles of $\mathrm{O}_{3}(\mathrm{ppb})$ and standard deviations (horizontal lines). The region name and the number of stations, using brackets, are given above each plot. 


\section{Tables}

Table S1: The chemical trace species for the CB05 chemical mechanism included in gasphase tropospheric chemistry version of NMMB/BSC-CTM.

\begin{tabular}{|c|c|c|c|}
\hline Species name & Description & Species name & Description \\
\hline $\mathrm{NO}$ & Nitric oxide & $\mathrm{SO}_{2}$ & Sulfur dioxide \\
\hline $\mathrm{NO}_{2}$ & Nitrogen dioxide & $\mathrm{MEO}_{2}$ & Methylperoxy radical \\
\hline $\mathrm{O}_{3}$ & Ozone & $\mathrm{MEOH}$ & Methanol \\
\hline $\mathrm{O}^{1} \mathrm{D}$ & Oxygen atom in the $\mathrm{O}^{1}(\mathrm{D})$ electronic state & FACD & Formic acid \\
\hline $\mathrm{OH}$ & Hydroxyl radical & ETHA & Ethane \\
\hline $\mathrm{HO}_{2}$ & Hydroperoxy radical & $\mathrm{ROOH}$ & Higher organic peroxide \\
\hline $\mathrm{NO}_{3}$ & Nitrate radical & PACD & Peroxyacetic and higher peroxycarboxylic acids \\
\hline $\mathrm{N}_{2} \mathrm{O}_{5}$ & Dinitrogen pentoxide & PAR & Paraffin carbon bond (C-C) \\
\hline $\mathrm{HONO}$ & Nitrous acid & ROR & Secondary alkoxy radical \\
\hline $\mathrm{HNO}_{3}$ & Nitric acid & ETH & Ethene \\
\hline PNA & Peroxynitric acid $\left(\mathrm{HNO}_{4}\right)$ & OLE & Terminal olefin carbon bond $(\mathrm{R}-\mathrm{C}=\mathrm{C})$ \\
\hline $\mathrm{CO}$ & Carbon monoxide & IOLE & Internal olefin carbon bond $(\mathrm{R}-\mathrm{C}=\mathrm{C}-\mathrm{R})$ \\
\hline FORM & Formaldehyde & ISOP & Isoprene \\
\hline $\mathrm{C}_{2} \mathrm{O}_{3}$ & Acetylperoxy radical & & \\
\hline PAN & Peroxyacetyl nitrate & TERP & Terpene \\
\hline ALDX & Propionaldehyde and higher aldehydes & TOL & Toluene and other monoalkyl aromatics \\
\hline $\mathrm{CXO}_{3}$ & C3 and higher acylperoxy radicals & XYL & Xylene and other polyalkyl aromatics \\
\hline PANX & $\mathrm{C} 3$ and higher peroxyacyl nitrates & CRES & Cresol and higher molecular weight phenols \\
\hline $\mathrm{XO}_{2}$ & $\begin{array}{l}\mathrm{NO} \text { to } \mathrm{NO}_{2} \text { conversion from alkylperoxy }\left(\mathrm{RO}_{2}\right) \text { radi- } \\
\text { cal }\end{array}$ & $\mathrm{TO}_{2}$ & Toluene-hydroxyl radical adduct \\
\hline $\mathrm{XO}_{2} \mathrm{~N}$ & $\begin{array}{l}\text { NO to organic nitrate conversion from alkylperoxy } \\
\left(\mathrm{RO}_{2}\right) \text { radical }\end{array}$ & OPEN & Aromatic ring opening product \\
\hline NTR & Organic nitrate $\left(\mathrm{RNO}_{3}\right)$ & $\mathrm{CRO}$ & Methylphenoxy radical \\
\hline ETOH & Ethanol & MGLY & Methylglyoxal and other aromatic products \\
\hline SULF & Sulfuric acid (gaseous) & & \\
\hline
\end{tabular}


Table S2: The gas-phase CB05 chemical mechanism reactions applied in the NMMB/BSC-CTM. The first column describes the reactants, the second the products and the third displays the coefficients to compute the full rate expressions for each reaction.

\begin{tabular}{|c|c|c|}
\hline Reactants & Products & Rate expression \\
\hline $\mathrm{O}+\mathrm{O}_{2}+\mathrm{M}$ & $\rightarrow \quad \mathrm{O}_{3}+\mathrm{M}$ & $6.0 \mathrm{E}-34 *(300 / \mathrm{T})^{2.4}$ \\
\hline $\mathrm{O}_{3}+\mathrm{NO}$ & $\rightarrow \quad \mathrm{NO}_{2}$ & 3.0E-12*exp(T/1500) \\
\hline $\mathrm{O}+\mathrm{NO}_{2}$ & $\rightarrow \quad \mathrm{NO}$ & $5.6 \mathrm{E}-12 * \exp (180 / \mathrm{T})$ \\
\hline $\mathrm{O}+\mathrm{NO}_{2}$ & $\rightarrow \quad \mathrm{NO}_{3}$ & $\begin{array}{l}\mathrm{K}_{0}=2.5 \mathrm{E}-31 * \exp (300 / \mathrm{T})^{1.8} \\
\mathrm{~K}_{\infty}=2.2 \mathrm{E}-11 * \exp (300 / \mathrm{T})^{0.7}\end{array}$ \\
\hline $\mathrm{O}+\mathrm{NO}$ & $\rightarrow \quad \mathrm{NO}_{2}$ & $\begin{array}{l}\mathrm{K}_{0}=9.0 \mathrm{E}-32 * \exp (300 / \mathrm{T})^{1.5} \\
\mathrm{~K}_{\infty}=3.0 \mathrm{E}-11\end{array}$ \\
\hline $\mathrm{NO}_{2}+\mathrm{O}_{3}$ & $\rightarrow \quad \mathrm{NO}_{3}$ & $1.2 \mathrm{E}-13 * \exp (\mathrm{T} / 2450)$ \\
\hline $\mathrm{O}\left({ }^{1}\right) \mathrm{D}+\mathrm{M}$ & $\rightarrow \quad \mathrm{O}+\mathrm{M}$ & $2.1 \mathrm{E}-11 * \exp (102 / \mathrm{T})$ \\
\hline $\mathrm{O}\left({ }^{1}\right) \mathrm{D}+\mathrm{H}_{2} \mathrm{O}$ & $\rightarrow \quad 2.000 * \mathrm{OH}$ & $2.2 \mathrm{E}-10$ \\
\hline $\mathrm{O}_{3}+\mathrm{OH}$ & $\rightarrow \quad \mathrm{HO}_{2}$ & $1.7 \mathrm{E}-12 * \exp (\mathrm{T} / 940)$ \\
\hline $\mathrm{O}_{3}+\mathrm{HO}_{2}$ & $\rightarrow \quad \mathrm{OH}$ & 1.0E-14*exp(T/490) \\
\hline $\mathrm{NO}_{3}+\mathrm{NO}$ & $2.000 * \mathrm{NO}_{2}$ & $1.5 \mathrm{E}-11 * \exp (170 / \mathrm{T})$ \\
\hline $\mathrm{NO}_{3}+\mathrm{NO}_{2}$ & $\rightarrow \quad \mathrm{NO}+\mathrm{NO}_{2}$ & $4.5 \mathrm{E}-14 * \exp (\mathrm{T} / 1260)$ \\
\hline $\mathrm{NO}_{3}+\mathrm{NO}_{2}$ & $\mathrm{~N}_{2} \mathrm{O}_{5}$ & $\begin{array}{l}\mathrm{K}_{0}=2.0 \mathrm{E}-30 *(300 / \mathrm{T})^{4.4} \\
\mathrm{~K}_{\infty}=1.4 \mathrm{E}-12 *(300 / \mathrm{T})^{0.7}\end{array}$ \\
\hline $\mathrm{N}_{2} \mathrm{O}_{5}+\mathrm{H}_{2} \mathrm{O}$ & $2.000 * \mathrm{HNO}_{3}$ & $2.5 \mathrm{E}-22$ \\
\hline $\mathrm{N}_{2} \mathrm{O}_{5}+\mathrm{H}_{2} \mathrm{O}+\mathrm{H}_{2} \mathrm{O}$ & $2.000 * \mathrm{HNO}_{3}$ & $1.8 \mathrm{E}-39$ \\
\hline $\mathrm{N}_{2} \mathrm{O}_{5}$ & $\mathrm{NO}_{3}+\mathrm{NO}_{2}$ & $\begin{array}{l}\mathrm{K}_{0}=1.0 \mathrm{E}-03 * \exp (11000 / \mathrm{T})^{3.5} \\
\mathrm{~K}_{\infty}=9.7 \mathrm{E}+14 * \exp (\mathrm{T} / 11080)^{0.1} \\
\mathrm{~F}_{c}=0.45 \\
\mathrm{n}=1.0\end{array}$ \\
\hline $\mathrm{NO}+\mathrm{NO}+\mathrm{O}_{2}$ & $2.000 * \mathrm{NO}_{2}$ & $3.3 \mathrm{E}-39 * \exp (530 / \mathrm{T})$ \\
\hline $\mathrm{NO}+\mathrm{NO}_{2}+\mathrm{H}_{2} \mathrm{O}$ & $2.000 * \mathrm{HONO}$ & $5.0 \mathrm{E}-40$ \\
\hline $\mathrm{NO}+\mathrm{OH}$ & HONO & $\begin{array}{l}\text { 7.0E-31*exp }(300 / \mathrm{T})^{2.6} \\
3.6 \mathrm{E}-11 * \exp (300 / \mathrm{T})-0.1\end{array}$ \\
\hline $\mathrm{OH}+\mathrm{HONO}$ & $\rightarrow \quad \mathrm{NO}_{2}$ & $1.8 \mathrm{E}-11 * \exp (\mathrm{T} / 390)$ \\
\hline $\mathrm{HONO}+\mathrm{HONO}$ & $\rightarrow \quad \mathrm{NO}+\mathrm{NO}_{2}$ & $1.0 \mathrm{E}-20$ \\
\hline $\mathrm{NO}_{2}+\mathrm{OH}$ & $\rightarrow \quad \mathrm{HNO}_{3}$ & $\begin{array}{l}\mathrm{K}_{0}=2.0 \mathrm{E}-30 * \exp (300 / \mathrm{T})^{3.0} \\
\mathrm{~K}_{\infty}=2.5 \mathrm{E}-11\end{array}$ \\
\hline $\mathrm{OH}+\mathrm{HNO}_{3}$ & $\mathrm{NO}_{3}$ & $\begin{array}{l}\mathrm{K}_{0}=2.4 \mathrm{E}-14 * \exp (460 / \mathrm{T}) \\
\mathrm{K}_{2}=2.7 \mathrm{E}-17 * \exp (2199 / \mathrm{T}) \\
\mathrm{K}_{3}=6.5 \mathrm{E}-34 * \exp (1335 / \mathrm{T})\end{array}$ \\
\hline $\mathrm{HO}_{2}+\mathrm{NO}$ & $\mathrm{OH}+\mathrm{NO}_{2}$ & $\mathrm{~K}_{0}=3.5 \mathrm{E}-12 * \exp (250 / \mathrm{T})$ \\
\hline $\mathrm{HO}_{2}+\mathrm{NO}_{2}$ & PNA & $\begin{array}{l}\mathrm{K}_{0}=1.8 \mathrm{E}-31 * \exp (300 / \mathrm{T})^{3.2} \\
\mathrm{~K}_{\infty}=4.7 \mathrm{E}-12 \\
\mathrm{~F}_{c}=0.6\end{array}$ \\
\hline PNA & $\rightarrow \quad \mathrm{HO}_{2}+\mathrm{NO}_{2}$ & $\begin{array}{l}\mathrm{K}_{0}=4.1 \mathrm{E}-5^{*} \exp (\mathrm{T} / 10650) \\
\mathrm{K}_{\infty}=4.8 \mathrm{E} 15^{*} \exp (\mathrm{T} / 11170) \\
\mathrm{F}_{c}=0.6\end{array}$ \\
\hline $\mathrm{OH}+\mathrm{PNA}$ & $\rightarrow \quad \mathrm{NO}_{2}$ & $1.3 \mathrm{E}-12 * \exp (380 / \mathrm{T})$ \\
\hline $\mathrm{HO}_{2}+\mathrm{HO}_{2}$ & $\rightarrow \quad \mathrm{H}_{2} \mathrm{O}_{2}$ & $\begin{array}{l}\mathrm{K}_{1}=2.3 \mathrm{E}-13 * \exp (600 / \mathrm{T}) \\
\mathrm{K}_{2}=1.7 \mathrm{E}-33^{*} \exp (1000 / \mathrm{T})\end{array}$ \\
\hline $\mathrm{HO}_{2}+\mathrm{HO}_{2}+\mathrm{H}_{2} \mathrm{O}$ & $\rightarrow \quad \mathrm{H}_{2} \mathrm{O}_{2}$ & $\begin{array}{l}\mathrm{K}_{1}=3.22 \mathrm{E}-34 * \exp (2800 / \mathrm{T}) \\
\mathrm{K}_{2}=2.38 \mathrm{E}-54 * \exp (3200 / \mathrm{T})\end{array}$ \\
\hline
\end{tabular}


Table S2: Continued from previous page

\begin{tabular}{|c|c|c|c|}
\hline Reactants & & Products & Rate expression \\
\hline $\mathrm{OH}+\mathrm{H}_{2} \mathrm{O}_{2}$ & $\rightarrow$ & $\mathrm{HO}_{2}$ & $2.9 \mathrm{E}-12 * \exp (\mathrm{T} / 160)$ \\
\hline $\mathrm{O}^{1} \mathrm{D}+\mathrm{H}_{2}$ & $\rightarrow$ & $\mathrm{OH}+\mathrm{HO}_{2}$ & $1.1 \mathrm{E}-10$ \\
\hline $\mathrm{OH}+\mathrm{H}_{2}$ & $\rightarrow$ & $\mathrm{HO}_{2}$ & $5.5 \mathrm{E}-12 * \exp (\mathrm{T} / 2000)$ \\
\hline $\mathrm{OH}+\mathrm{O}$ & $\rightarrow$ & $\mathrm{HO}_{2}$ & $2.2 \mathrm{E}-11 * \exp (120 / \mathrm{T})$ \\
\hline $\mathrm{OH}+\mathrm{OH}$ & $\rightarrow$ & $\mathrm{O}$ & $4.2 \mathrm{E}-12 * \exp (\mathrm{T} / 240)$ \\
\hline $\mathrm{OH}+\mathrm{OH}$ & $\rightarrow$ & $\mathrm{H}_{2} \mathrm{O}_{2}$ & $\begin{array}{l}\mathrm{K}_{0}=6.9 \mathrm{E}-31 * \exp (300 / \mathrm{T})^{1.0} \\
\mathrm{~K}_{\infty}=2.6 \mathrm{E}-11\end{array}$ \\
\hline $\mathrm{OH}+\mathrm{HO}_{2}$ & $\rightarrow$ & & $4.8 \mathrm{E}-11 * \exp (250 / \mathrm{T})$ \\
\hline $\mathrm{HO}_{2}+\mathrm{O}$ & $\rightarrow$ & $\mathrm{OH}$ & $3.0 \mathrm{E}-11 * \exp (200 / \mathrm{T})$ \\
\hline $\mathrm{H}_{2} \mathrm{O}_{2}+\mathrm{O}$ & $\rightarrow$ & $\mathrm{OH}+\mathrm{HO}_{2}$ & 1.4E-12*exp(-2000/T) \\
\hline $\mathrm{NO}_{3}+\mathrm{O}$ & $\rightarrow$ & $\mathrm{NO}_{2}$ & $1.0 \mathrm{E}-11$ \\
\hline $\mathrm{NO}_{3}+\mathrm{OH}$ & $\rightarrow$ & $\mathrm{HO}_{2}+\mathrm{NO}_{2}$ & $2.2 \mathrm{E}-11$ \\
\hline $\mathrm{NO}_{3}+\mathrm{HO}_{2}$ & $\rightarrow$ & $\mathrm{HNO}_{3}$ & $3.5 \mathrm{E}-12$ \\
\hline $\mathrm{NO}_{3}+\mathrm{O}_{3}$ & $\rightarrow$ & $\mathrm{NO}_{2}$ & $1.0 \mathrm{E}-17$ \\
\hline $\mathrm{NO}_{3}+\mathrm{NO}_{3}$ & $\rightarrow$ & $2.000 * \mathrm{NO}_{2}$ & $8.5 \mathrm{E}-13 * \exp (\mathrm{T} / 2450)$ \\
\hline $\mathrm{XO}_{2}+\mathrm{NO}$ & $\vec{b}$ & $\mathrm{NO}_{2}$ & $2.6 \mathrm{E}-12 * \exp (365 / \mathrm{T})$ \\
\hline $\mathrm{XO}_{2} \mathrm{~N}+\mathrm{NO}$ & $\rightarrow$ & NTR & $2.6 \mathrm{E}-12 * \exp (365 / \mathrm{T})$ \\
\hline $\mathrm{XO}_{2}+\mathrm{HO}_{2}$ & $\rightarrow$ & $\mathrm{ROOH}$ & $7.5 \mathrm{E}-13 * \exp (700 / \mathrm{T})$ \\
\hline $\mathrm{XO}_{2} \mathrm{~N}+\mathrm{HO}_{2}$ & $\rightarrow$ & $\mathrm{ROOH}$ & $7.5 \mathrm{E}-13 * \exp (700 / \mathrm{T})$ \\
\hline $\mathrm{XO}_{2}+\mathrm{XO}_{2}$ & $\rightarrow$ & & $6.8 \mathrm{E}-14$ \\
\hline $\mathrm{XO}_{2} \mathrm{~N}+\mathrm{XO}_{2} \mathrm{~N}$ & $\rightarrow$ & & $6.8 \mathrm{E}-14$ \\
\hline $\mathrm{XO}_{2}+\mathrm{XO}_{2} \mathrm{~N}$ & $\rightarrow$ & & $6.8 \mathrm{E}-14$ \\
\hline $\mathrm{NTR}+\mathrm{OH}$ & $\rightarrow$ & 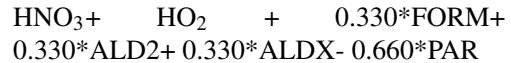 & $5.9 \mathrm{E}-13 * \exp (360 / \mathrm{T})$ \\
\hline $\mathrm{ROOH}+\mathrm{OH}$ & $\rightarrow$ & $\mathrm{XO}_{2}+0.500 * \mathrm{ALD} 2+0.500 *$ ALDX & $3.01 \mathrm{E}-12 * \exp (190 / \mathrm{T})$ \\
\hline $\mathrm{OH}+\mathrm{CO}$ & $\rightarrow$ & $\mathrm{HO}_{2}$ & $\begin{array}{l}\mathrm{K}_{1}=1.44 \mathrm{E}-13 \\
\mathrm{~K}_{2}=3.43 \mathrm{E}-33\end{array}$ \\
\hline $\mathrm{OH}+\mathrm{CH} 4$ & $\rightarrow$ & $\mathrm{MEO}_{2}$ & $2.45 \mathrm{E}-12 * \exp (\mathrm{T} / 1775)$ \\
\hline $\mathrm{MEO}_{2}+\mathrm{NO}$ & $\rightarrow$ & $\mathrm{FORM}+\mathrm{HO}_{2}+\mathrm{NO}_{2}$ & $2.8 \mathrm{E}-12 * \exp (300 / \mathrm{T})$ \\
\hline $\mathrm{MEO}_{2}+\mathrm{HO}_{2}$ & $\rightarrow$ & MEPX & $4.1 \mathrm{E}-13 * \exp (750 / \mathrm{T})$ \\
\hline $\mathrm{MEO}_{2}+\mathrm{MEO}_{2}$ & $\rightarrow$ & $1.370 * \mathrm{FORM}+0.740 * \mathrm{HO}_{2}+0.630 * \mathrm{MEOH}$ & $9.5 \mathrm{E}-14 * \exp (390 / \mathrm{T})$ \\
\hline $\mathrm{MEPX}+\mathrm{OH}$ & $\rightarrow$ & $0.700 * \mathrm{MEO}_{2}+0.300 * \mathrm{XO}_{2}+0.300 * \mathrm{HO}_{2}$ & $3.8 \mathrm{E}-12 * \exp (200 / \mathrm{T})$ \\
\hline $\mathrm{MEOH}+\mathrm{OH}$ & $\rightarrow$ & $\mathrm{FORM}+\mathrm{HO}_{2}$ & $7.3 \mathrm{E}-12 * \exp (\mathrm{T} / 620)$ \\
\hline $\mathrm{FORM}+\mathrm{OH}$ & $\rightarrow$ & $\mathrm{HO}_{2}+\mathrm{CO}$ & $9.0 \mathrm{E}-12$ \\
\hline $\mathrm{FORM}+\mathrm{O}$ & $\rightarrow$ & $\mathrm{OH}+\mathrm{HO}_{2}+\mathrm{CO}$ & $3.4 \mathrm{E}-11 * \exp (\mathrm{T} / 1600)$ \\
\hline $\mathrm{FORM}+\mathrm{NO}_{3}$ & $\rightarrow$ & $\mathrm{HNO}_{3}+\mathrm{HO}_{2}+\mathrm{CO}$ & $5.8 \mathrm{E}-16$ \\
\hline $\mathrm{FORM}+\mathrm{HO}_{2}$ & $\rightarrow$ & $\mathrm{HCO}_{3}$ & $9.7 \mathrm{E}-15 * \exp (625 / \mathrm{T})$ \\
\hline $\mathrm{HCO}_{3}$ & $\rightarrow$ & $\mathrm{FORM}+\mathrm{HO}_{2}$ & $2.4 \mathrm{E}+12 * \exp (\mathrm{T} / 7000)$ \\
\hline $\mathrm{HCO}_{3}+\mathrm{NO}$ & $\vec{\rightarrow}$ & $\mathrm{FACD}+\mathrm{NO}_{2}+\mathrm{HO}_{2}$ & $5.6 \mathrm{E}-12$ \\
\hline $\mathrm{HCO}_{3}+\mathrm{HO}_{2}$ & $\rightarrow$ & MEPX & $5.6 \mathrm{E}-15 * \exp (2300 / \mathrm{T})$ \\
\hline $\mathrm{FACD}+\mathrm{OH}$ & $\rightarrow$ & $\mathrm{HO}_{2}$ & 4.0E-13 \\
\hline $\mathrm{ALD} 2+\mathrm{O}$ & $\rightarrow$ & $\mathrm{C}_{2} \mathrm{O}_{3}+\mathrm{OH}$ & $1.8 \mathrm{E}-11 * \exp (\mathrm{T} / 1100)$ \\
\hline $\mathrm{ALD} 2+\mathrm{OH}$ & $\rightarrow$ & $\mathrm{C}_{2} \mathrm{O}_{3}$ & $5.6 \mathrm{E}-12 * \exp (270 / \mathrm{T})$ \\
\hline $\mathrm{ALD} 2+\mathrm{NO}_{3}$ & $\rightarrow$ & $\mathrm{C}_{2} \mathrm{O}_{3}+\mathrm{HNO}_{3}$ & $1.4 \mathrm{E}-12 * \exp (\mathrm{T} / 1900)$ \\
\hline $\mathrm{C}_{2} \mathrm{O}_{3}+\mathrm{NO}$ & $\rightarrow$ & $\mathrm{MEO}_{2}+\mathrm{NO}_{2}$ & $8.1 \mathrm{E}-12 * \exp (270 / \mathrm{T})$ \\
\hline PAN & $\rightarrow$ & $\mathrm{C}_{2} \mathrm{O}_{3}+\mathrm{NO}_{2}$ & $\begin{array}{l}\mathrm{K}_{0}=4.9 \mathrm{E}-3 * \exp (12100 / \mathrm{T}) \\
\mathrm{K}_{\infty}=5.4 \mathrm{E} 16^{*} \exp (\mathrm{T} / 13830) \\
\mathrm{F}_{c}=0.3\end{array}$ \\
\hline $\mathrm{C}_{2} \mathrm{O}_{3}+\mathrm{HO}_{2}$ & $\rightarrow$ & $0.800 * \mathrm{PACD}+0.200 * \mathrm{AACD}+0.200 * \mathrm{O}_{3}$ & 4.3E-13*exp $(1040 / \mathrm{T})$ \\
\hline $\mathrm{C}_{2} \mathrm{O}_{3}+\mathrm{MEO}_{2}$ & $\rightarrow$ & $\begin{array}{l}0.900 * \mathrm{MEO}_{2}+ \\
0.100 * \mathrm{AACD}\end{array}$ & $2.0 \mathrm{E}-12 * \exp (500 / \mathrm{T})$ \\
\hline
\end{tabular}


Table S2: Continued from previous page

\begin{tabular}{|c|c|c|c|}
\hline Reactants & & Products & Rate expression \\
\hline $\mathrm{C}_{2} \mathrm{O}_{3}+\mathrm{XO}_{2}$ & $\rightarrow$ & $0.900 * \mathrm{MEO}_{2}+0.100 * \mathrm{AACD}$ & $4.4 \mathrm{E}-13^{*} \exp (1070 / \mathrm{T})$ \\
\hline $\mathrm{C}_{2} \mathrm{O}_{3}+\mathrm{C}_{2} \mathrm{O}_{3}$ & $\rightarrow$ & $2.000 * \mathrm{MEO}_{2}$ & $2.9 \mathrm{E}-12 * \exp (500 / \mathrm{T})$ \\
\hline $\mathrm{PACD}+\mathrm{OH}$ & $\rightarrow$ & $\mathrm{C}_{2} \mathrm{O}_{3}$ & $4.0 \mathrm{E}-13 * \exp (200 / \mathrm{T})$ \\
\hline $\mathrm{AACD}+\mathrm{OH}$ & $\rightarrow$ & $\mathrm{MEO}_{2}$ & $4.0 \mathrm{E}-13 * \exp (200 / \mathrm{T})$ \\
\hline $\mathrm{ALDX}+\mathrm{O}$ & $\rightarrow$ & $\mathrm{CXO}_{3}+\mathrm{OH}$ & $1.3 \mathrm{E}-11 * \exp (\mathrm{T} / 870)$ \\
\hline $\mathrm{ALDX}+\mathrm{OH}$ & $\rightarrow$ & $\mathrm{CXO}_{3}$ & $5.1 \mathrm{E}-12 * \exp (405 / \mathrm{T})$ \\
\hline $\mathrm{ALDX}+\mathrm{NO}_{3}$ & $\rightarrow$ & $\mathrm{CXO}_{3}+\mathrm{HNO}_{3}$ & $6.5 \mathrm{E}-15$ \\
\hline $\mathrm{CXO}_{3}+\mathrm{NO}$ & $\rightarrow$ & $\mathrm{ALD} 2+\mathrm{NO}_{2}+\mathrm{HO}_{2}+\mathrm{XO}_{2}$ & $6.7 \mathrm{E}-12 * \exp (340 / \mathrm{T})$ \\
\hline $\mathrm{CXO}_{3}+\mathrm{NO}_{2}$ & $\rightarrow$ & PANX & $\begin{array}{l}\mathrm{K}_{0}=2.7 \mathrm{E}-28 * \exp (300 / \mathrm{T})^{7.1} \\
\mathrm{~K}_{\infty}=1.2 \mathrm{E}-11 * \exp (300 / \mathrm{T})^{0.9} \\
\mathrm{~F}_{c}=0.3\end{array}$ \\
\hline PANX & $\rightarrow$ & $\mathrm{CXO}_{3}+\mathrm{NO}_{2}$ & \\
\hline $\mathrm{PANX}+\mathrm{OH}$ & $\rightarrow$ & $\mathrm{ALD} 2+\mathrm{NO}_{2}$ & $3.0 \mathrm{E}-13$ \\
\hline $\mathrm{CXO}_{3}+\mathrm{HO}_{2}$ & $\rightarrow$ & $0.800 * \mathrm{PACD}+0.200 * \mathrm{AACD}+0.200 * \mathrm{O}_{3}$ & $4.3 \mathrm{E}-13^{*} \exp (1040 / \mathrm{T})$ \\
\hline $\mathrm{CXO}_{3}+\mathrm{MEO}_{2}$ & $\rightarrow$ & 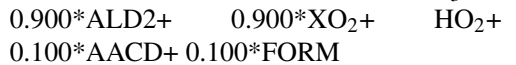 & $2.0 \mathrm{E}-12 * \exp (500 / \mathrm{T})$ \\
\hline $\mathrm{CXO}_{3}+\mathrm{XO}_{2}$ & $\rightarrow$ & $0.900 * \mathrm{ALD} 2+0.100 * \mathrm{AACD}$ & $4.4 \mathrm{E}-13^{*} \exp (1070 / \mathrm{T})$ \\
\hline $\mathrm{CXO}_{3}+\mathrm{CXO}_{3}$ & $\rightarrow$ & $2.000 * \mathrm{ALD} 2+2.000 * \mathrm{XO}_{2}+2.000 * \mathrm{HO}_{2}$ & $2.9 \mathrm{E}-12 * \exp (500 / \mathrm{T})$ \\
\hline $\mathrm{CXO}_{3}+\mathrm{C}_{2} \mathrm{O}_{3}$ & $\rightarrow$ & $\begin{array}{l}\mathrm{MEO}_{2}+\mathrm{XO}_{2}+\mathrm{HO}_{2}+\mathrm{ALD} 2 \\
0.870 * \mathrm{XO}_{2}+0.130 * \mathrm{XO}_{2} \mathrm{~N}+0.110 * \mathrm{HO}_{2}+\end{array}$ & $2.9 \mathrm{E}-12 * \exp (500 / \mathrm{T})$ \\
\hline $\mathrm{PAR}+\mathrm{OH}$ & $\rightarrow$ & 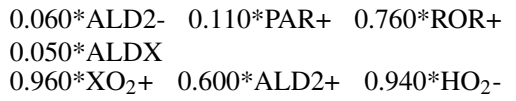 & $8.1 \mathrm{E}-13$ \\
\hline ROR & $\rightarrow$ & $\begin{array}{l}2.100 * \mathrm{PAR}+0.040 * \mathrm{XO}_{2} \mathrm{~N}+0.020 * \mathrm{ROR}+ \\
0.500 * \mathrm{ALDX}\end{array}$ & $1 . \mathrm{E}+15 * \exp (\mathrm{T} / 8000)$ \\
\hline ROR & $\rightarrow$ & $\mathrm{HO}_{2}$ & $1.6 \mathrm{E}+3$ \\
\hline $\mathrm{ROR}+\mathrm{NO}_{2}$ & $\rightarrow$ & $\begin{array}{l}\text { NTR } \\
0.200 * A L D 2+0.300 * A L D X+0.300 * \mathrm{HO}_{2}+\end{array}$ & $1.5 \mathrm{E}-11$ \\
\hline $\mathrm{O}+\mathrm{OLE}$ & $\rightarrow$ & 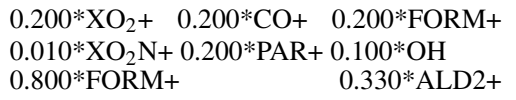 & 1.E-11*exp(T/280) \\
\hline $\mathrm{OH}+\mathrm{OLE}$ & $\rightarrow$ & $\begin{array}{lr}0.620 * \mathrm{ALDX}+ & 0.800 * \mathrm{XO}_{2}+0.950 * \mathrm{HO}_{2-} \\
0.700 * \mathrm{PAR} & \\
0.180 * \mathrm{ALD} 2+ & 0.740 * \mathrm{FORM}+\end{array}$ & $3.2 \mathrm{E}-11$ \\
\hline $\mathrm{O}_{3}+\mathrm{OLE}$ & $\rightarrow$ & $\begin{array}{l}0.320 * \mathrm{ALDX}+0.220 * \mathrm{XO}_{2}+0.100 * \mathrm{OH}+ \\
0.330 * \mathrm{CO}+0.440 * \mathrm{HO}_{2}-1.000 * \mathrm{PAR}\end{array}$ & $6.5 \mathrm{E}-15^{*} \exp (\mathrm{T} / 1900)$ \\
\hline $\mathrm{NO}_{3}+\mathrm{OLE}$ & $\rightarrow$ & $\begin{array}{l}\mathrm{NO}_{2}+\mathrm{FORM}+0.910 * \mathrm{XO}_{2}+0.090 * \mathrm{XO}_{2} \mathrm{~N}+ \\
0.560 * \mathrm{ALDX}+0.350 * \mathrm{ALD} 2-1.000 * \mathrm{PAR}\end{array}$ & $7.0 \mathrm{E}-13^{*} \exp (\mathrm{T} / 2160)$ \\
\hline $\mathrm{O}+\mathrm{ETH}$ & $\rightarrow$ & $\begin{array}{l}\mathrm{FORM}+1.700 * \mathrm{HO}_{2}+\mathrm{CO}+0.700 * \mathrm{XO}_{2}+ \\
0.300 * \mathrm{OH}\end{array}$ & $1.04 \mathrm{E}-11 * \exp (\mathrm{T} / 792)$ \\
\hline $\mathrm{OH}+\mathrm{ETH}$ & $\rightarrow$ & $\mathrm{XO}_{2}+1.560 * \mathrm{FORM}+0.220 * \mathrm{ALDX}+\mathrm{HO}_{2}$ & $\begin{array}{l}\mathrm{K}_{0}=1.0 \mathrm{E}-28 * \exp (300 / \mathrm{T})^{0.8} \\
\mathrm{~K}_{\infty}=8.8 \mathrm{E}-12\end{array}$ \\
\hline $\mathrm{O}_{3}+\mathrm{ETH}$ & $\rightarrow$ & $\begin{array}{lr}\mathrm{FORM}+ & 0.630 * \mathrm{CO}+ \\
0.130 * \mathrm{OH}+ & 0.370 * \mathrm{FACD}\end{array} \quad 0.130 * \mathrm{HO}_{2}+$ & $1.2 \mathrm{E}-14 * \exp (\mathrm{T} / 2630)$ \\
\hline $\mathrm{NO}_{3}+\mathrm{ETH}$ & $\rightarrow$ & $\mathrm{NO}_{2}+\mathrm{XO}_{2}+2.0 * \mathrm{FORM}$ & $3.3 \mathrm{E}-12 * \exp (\mathrm{T} / 2880)$ \\
\hline $\mathrm{IOLE}+\mathrm{O}$ & $\rightarrow$ & $\begin{array}{l}1.240 * \mathrm{ALD} 2+0.660 * \mathrm{ALDX}+0.100 * \mathrm{HO}_{2}+ \\
0.100 * \mathrm{XO}_{2}+0.100 * \mathrm{CO}+0.100 * \mathrm{PAR}\end{array}$ & $2.3 \mathrm{E}-11$ \\
\hline $\mathrm{IOLE}+\mathrm{OH}$ & $\rightarrow$ & $\begin{array}{l}1.300 * \mathrm{ALD} 2+0.700 * \mathrm{ALDX}+\mathrm{HO}_{2}+\mathrm{XO}_{2} \\
0.650 * \mathrm{ALD} 2+0.350 * \mathrm{ALDX}+\end{array}$ & $1.0 \mathrm{E}-11 * \exp (550 / \mathrm{T})$ \\
\hline $\mathrm{IOLE}+\mathrm{O}_{3}$ & $\rightarrow$ & $\begin{array}{l}0.250 * \mathrm{FORM}+0.250 * \mathrm{CO}+0.500 * \mathrm{O} \\
+0.500 * \mathrm{OH}+0.500 * \mathrm{HO}_{2}\end{array}$ & $8.4 \mathrm{E}-15^{*} \exp (\mathrm{T} / 1100)$ \\
\hline
\end{tabular}


Table S2: Continued from previous page

\begin{tabular}{|c|c|c|c|}
\hline Reactants & & Products & Rate expression \\
\hline $\mathrm{IOLE}+\mathrm{NO}_{3}$ & $\rightarrow$ & $1.180^{*} \mathrm{ALD} 2+0.640^{*} \mathrm{ALDX}+\mathrm{HO}_{2}+\mathrm{NO}_{2}$ & $9.6 \mathrm{E}-13 * \exp (\mathrm{T} / 270)$ \\
\hline $\mathrm{TOL}+\mathrm{OH}$ & $\rightarrow$ & $\begin{array}{l}0.440 * \mathrm{HO}_{2}+0.080 * \mathrm{XO}_{2}+0.360 * \mathrm{CRES}+ \\
0.560 * \mathrm{TO}_{2}+0.765 * \mathrm{TOLRO}_{2}\end{array}$ & $1.8 \mathrm{E}-12 * \exp (355 / \mathrm{T})$ \\
\hline $\mathrm{TO}_{2}+\mathrm{NO}$ & $\rightarrow$ & $\begin{array}{l}0.900 * \mathrm{NO}_{2}+0.900 * \mathrm{HO}_{2}+0.900 * \mathrm{OPEN}+ \\
0.100 * \mathrm{NTR}\end{array}$ & $8.1 \mathrm{E}-12$ \\
\hline $\mathrm{TO}_{2}$ & $\rightarrow$ & $\mathrm{CRES}+\mathrm{HO}_{2}$ & 4.2 \\
\hline $\mathrm{OH}+\mathrm{CRES}$ & $\rightarrow$ & $\begin{array}{l}0.400 * \mathrm{CRO}+0.600 * \mathrm{XO}_{2}+0.600 * \mathrm{HO}_{2}+ \\
0.300 * \text { OPEN }\end{array}$ & 4.1E-11 \\
\hline $\mathrm{CRES}+\mathrm{NO}_{3}$ & $\rightarrow$ & $\mathrm{CRO}+\mathrm{HNO}_{3}$ & $2.2 \mathrm{E}-11$ \\
\hline $\mathrm{CRO}+\mathrm{NO}_{2}$ & $\rightarrow$ & NTR & $1.4 \mathrm{E}-11$ \\
\hline $\mathrm{CRO}+\mathrm{HO}_{2}$ & $\rightarrow$ & CRES & $5.5 \mathrm{E}-12$ \\
\hline $\mathrm{OPEN}+\mathrm{OH}$ & $\rightarrow$ & $\begin{array}{l}\mathrm{XO}_{2}+2.000 * \mathrm{CO}+2.000 * \mathrm{HO}_{2}+\mathrm{C}_{2} \mathrm{O}_{3}+ \\
\text { FORM } \\
0.030 * \mathrm{ALDX}+0.620 * \mathrm{C}_{2} \mathrm{O}_{3}+\end{array}$ & 3.0E-11 \\
\hline $\mathrm{OPEN}+\mathrm{O}_{3}$ & $\rightarrow$ & $\begin{array}{l}0.700 * \mathrm{FORM}+0.030 * \mathrm{XO}_{2}+0.690 * \mathrm{CO}+ \\
0.080 * \mathrm{OH}+0.760 * \mathrm{HO}_{2}+0.200 * \mathrm{MGLY} \\
0.700 * \mathrm{HO}_{2}+0.500 * \mathrm{XO}_{2}+0.200 * \mathrm{CRES}+\end{array}$ & $5.4 \mathrm{E}-17 * \exp (\mathrm{T} / 500)$ \\
\hline $\mathrm{OH}+\mathrm{XYL}$ & $\rightarrow$ & $\begin{array}{l}0.800 * \mathrm{MGLY}^{+} 1.100 * \mathrm{PAR}+0.300 * \mathrm{TO}_{2}+ \\
0.804 * \mathrm{XYLRO}_{2}\end{array}$ & $1.7 \mathrm{E}-11 * \exp (116 / \mathrm{T})$ \\
\hline $\mathrm{OH}+\mathrm{MGLY}$ & $\rightarrow$ & $\mathrm{XO}_{2}+\mathrm{C}_{2} \mathrm{O}_{3}$ & $1.8 \mathrm{E}-11$ \\
\hline $\mathrm{O}+\mathrm{ISOP}$ & $\rightarrow$ & $\begin{array}{l}0.750 * \text { ISPD }+0.500 * \mathrm{FORM}+0.250 * \mathrm{XO}_{2} \\
+0.250 * \mathrm{HO}_{2}+0.250 * \mathrm{CXO}_{3}+0.250 * \mathrm{PAR}\end{array}$ & 3.6E-11 \\
\hline $\mathrm{OH}+\mathrm{ISOP}$ & $\rightarrow$ & 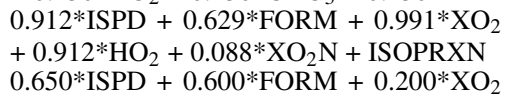 & $2.54 \mathrm{E}-11 * \exp (407.6 / \mathrm{T})$ \\
\hline $\mathrm{O}_{3}+\mathrm{ISOP}$ & $\rightarrow$ & $\begin{array}{l}+0.066 * \mathrm{HO}_{2}+0.266 * \mathrm{OH}+0.200 * \mathrm{CXO}_{3}+ \\
0.150 * \mathrm{ALDX}+0.350 * \mathrm{PAR}+0.066 * \mathrm{CO} \\
0.200 * \mathrm{ISPD}+0.800 * \mathrm{NTR}+\mathrm{XO}_{2}+\end{array}$ & $7.86 \mathrm{E}-15^{*} \exp (\mathrm{T} / 1912)$ \\
\hline $\mathrm{NO}_{3}+\mathrm{ISOP}$ & $\rightarrow$ & $\begin{array}{l}0.800 * \mathrm{HO}_{2}+0.200 * \mathrm{NO}_{2}+0.800 * \mathrm{ALDX}+ \\
2.400 * \mathrm{PAR} \\
1.565 * \mathrm{PAR}+0.167 * \mathrm{FORM}+0.713 * \mathrm{XO}_{2}+\end{array}$ & $3.03 \mathrm{E}-12 * \exp (\mathrm{T} / 448)$ \\
\hline $\mathrm{OH}+\mathrm{ISPD}$ & $\rightarrow$ & $\begin{array}{l}0.503 * \mathrm{HO}_{2}+0.334 * \mathrm{CO}+0.168 * \mathrm{MGLY}+ \\
0.252 * \mathrm{ALD} 2+0.210 * \mathrm{C}_{2} \mathrm{O}_{3}+0.250 * \mathrm{CXO}_{3} \\
+0.120 * \mathrm{ALDX}\end{array}$ & $3.36 \mathrm{E}-11$ \\
\hline $\mathrm{O}_{3}+\mathrm{ISPD}$ & $\rightarrow$ & $\begin{array}{l}0.114 * \mathrm{C}_{2} \mathrm{O}_{3}+0.150 * \mathrm{FORM}+ \\
0.850 * \mathrm{MGLY}^{+}+0.154 * \mathrm{HO}_{2}+0.268 * \mathrm{OH}+ \\
0.064 * \mathrm{XO}_{2}+0.020 * \mathrm{ALD} 2+0.360 * \mathrm{PAR}+ \\
0.225 * \mathrm{CO} \\
0.357 * \mathrm{ALDX}+0.282 * \mathrm{FORM}+1.282 * \mathrm{PAR}\end{array}$ & 7.1E-18 \\
\hline $\mathrm{NO}_{3}+\mathrm{ISPD}$ & $\rightarrow$ & $\begin{array}{l}+0.925 * \mathrm{HO}_{2}+0.643 * \mathrm{CO}+0.850 * \mathrm{NTR}_{+}+ \\
0.075 * \mathrm{CXO}_{3}+0.075 * \mathrm{XO}_{2}+0.150 * \mathrm{HNO}_{3}\end{array}$ & $1.0 \mathrm{E}-15$ \\
\hline $\mathrm{TERP}+\mathrm{O}$ & $\rightarrow$ & $\begin{array}{l}0.150 * \mathrm{ALDX}^{+} 5.12 * \mathrm{PAR}+\mathrm{TRPRXN} \\
0.750 * \mathrm{HO}_{2}+1.250 * \mathrm{XO}_{2}+0.250 * \mathrm{XO}_{2} \mathrm{~N}+\end{array}$ & 3.6E-11 \\
\hline $\mathrm{TERP}+\mathrm{OH}$ & $\rightarrow$ & $\begin{array}{l}0.280 * \text { FORM }+1.66 * \text { PAR }+0.470 * \text { ALDX } \\
+ \text { TRPRXN }\end{array}$ & $1.5 \mathrm{E}-11 * \exp (449 / \mathrm{T})$ \\
\hline $\mathrm{TERP}+\mathrm{O}_{3}$ & $\rightarrow$ & $\begin{array}{l}0.570 * \mathrm{OH}+0.070 * \mathrm{HO}_{2}+0.760 * \mathrm{XO}_{2}+ \\
0.180 * \mathrm{XO}_{2} \mathrm{~N}+0.240 * \mathrm{FORM}+0.001 * \mathrm{CO}+ \\
7.000 * \mathrm{PAR}+0.210 * \mathrm{ALDX}+0.390 * \mathrm{CXO}_{3} \\
+ \text { TRPRXN }\end{array}$ & $1.2 \mathrm{E}-15^{*} \exp (\mathrm{T} / 821)$ \\
\hline $\mathrm{TERP}+\mathrm{NO}_{3}$ & $\rightarrow$ & $\begin{array}{l}0.470 * \mathrm{NO}_{2}+0.280 * \mathrm{HO}_{2}+1.030 * \mathrm{XO}_{2}+ \\
0.250 * \mathrm{XO}_{2} \mathrm{~N}+0.470 * \mathrm{ALDX}+0.530 * \mathrm{NTR} \\
+ \text { TRPRXN }\end{array}$ & $3.7 \mathrm{E}-12 * \exp (175 / \mathrm{T})$ \\
\hline
\end{tabular}


Table S2: Continued from previous page

\begin{tabular}{|c|c|c|c|}
\hline Reactants & & Products & Rate expression \\
\hline $\mathrm{SO}_{2}+\mathrm{OH}$ & $\rightarrow$ & $\mathrm{SULF}+\mathrm{HO}_{2}+\mathrm{SULRXN}$ & $\begin{array}{l}\mathrm{K}_{0}=3.0 \mathrm{E}-31 * \exp (300 / \mathrm{T})^{3.3} \\
\mathrm{~K}_{\infty}=1.5 \mathrm{E}-12\end{array}$ \\
\hline $\mathrm{OH}+\mathrm{ETOH}$ & $\rightarrow$ & $\begin{array}{l}\mathrm{HO}_{2}+0.900 * \mathrm{ALD} 2+0.050 * \mathrm{ALDX}+ \\
0.100 * \mathrm{FORM}+0.100 * \mathrm{XO}_{2}\end{array}$ & $6.9 \mathrm{E}-12 * \exp (\mathrm{T} / 230)$ \\
\hline $\mathrm{OH}+\mathrm{ETHA}$ & $\rightarrow$ & $\begin{array}{l}0.991 * \mathrm{ALD} 2+0.991 * \mathrm{XO}_{2}+0.009 * \mathrm{XO}_{2} \mathrm{~N} \\
+\mathrm{HO}_{2} \\
0.200 * \mathrm{ISPD}+0.800 * \mathrm{NTR}+\mathrm{XO}_{2}+\end{array}$ & $8.7 \mathrm{E}-12 * \exp (\mathrm{T} / 1070)$ \\
\hline $\mathrm{NO}_{2}+\mathrm{ISOP}$ & $\rightarrow$ & $\begin{array}{l}0.800 * \mathrm{HO}_{2}+0.200 * \mathrm{NO}+0.800 * \mathrm{ALDX}+ \\
2.400 * \mathrm{PAR}\end{array}$ & $1.5 \mathrm{E}-19$ \\
\hline
\end{tabular}


Table S3: Photolysis reactions applied in the NMMB/BSC-CTM

\begin{tabular}{lll}
\hline Reactants & & Products \\
\hline $\mathrm{NO}_{2}+\mathrm{hv}$ & $\rightarrow \mathrm{NO}+\mathrm{O}$ \\
$\mathrm{O}_{3}+\mathrm{hv}$ & $\rightarrow \mathrm{O}$ \\
$\mathrm{O}_{3}+\mathrm{hv}$ & $\rightarrow \mathrm{O}^{1} \mathrm{D}$ \\
$\mathrm{NO}_{3}+\mathrm{hv}$ & $\rightarrow \mathrm{NO}+\mathrm{O}$ \\
$\mathrm{NO}_{3}+\mathrm{hv}$ & $\rightarrow \mathrm{NO}$ \\
$\mathrm{HONO}+\mathrm{hv}$ & $\rightarrow \mathrm{NO}+\mathrm{OH}$ \\
$\mathrm{H}_{2} \mathrm{O}_{2}+\mathrm{hv}$ & $\rightarrow 2.000 * \mathrm{OH}$ \\
$\mathrm{PNA}+\mathrm{hv}$ & $\rightarrow 0.610 * \mathrm{HO}_{2}+0.610 * \mathrm{NO}_{2}+0.390 * \mathrm{OH}+$ \\
$\mathrm{HNO}+\mathrm{hv}$ & $\rightarrow 0.390 * \mathrm{NO}_{3}$ \\
$\mathrm{~N} 2 \mathrm{O} 5+\mathrm{hv}$ & $\rightarrow \mathrm{NO}_{2}+\mathrm{NO}_{3}$ \\
$\mathrm{NTR}+\mathrm{hv}$ & $\rightarrow \mathrm{NO}+\mathrm{HO}_{2}+0.330 * \mathrm{FORM}+0.330 * \mathrm{ALD} 2+$ \\
$\mathrm{FORM}+\mathrm{hv}$ & $\rightarrow 2.330 * \mathrm{ALDX}^{2}-0.660 * \mathrm{PAR}$ \\
$\mathrm{FORM}+\mathrm{hv}$ & $\rightarrow \mathrm{CO}_{2}+\mathrm{CO}$ \\
$\mathrm{ALD}+\mathrm{hv}$ & $\rightarrow \mathrm{MEO}_{2}+\mathrm{CO}+\mathrm{HO}_{2}$ \\
$\mathrm{PAN}+\mathrm{hv}$ & $\rightarrow \mathrm{C}_{2} \mathrm{O}_{3}+\mathrm{NO}_{2}$ \\
$\mathrm{PANX}+\mathrm{hv}$ & $\rightarrow \mathrm{CXO}_{3}+\mathrm{NO}_{2}$ \\
$\mathrm{PACD}+\mathrm{hv}$ & $\rightarrow \mathrm{MEO}_{2}+\mathrm{OH}$ \\
$\mathrm{ALDX}+\mathrm{hv}$ & $\rightarrow \mathrm{MEO}_{2}+\mathrm{CO}+\mathrm{HO}_{2}$ \\
\hline
\end{tabular}

\section{References}

W. M. Cox and J. A. Tikvart. A statistical procedure for determining the best performing air quality simulation model . Atmospheric Environment. Part A. General Topics, 24(9):2387 2395, 1990. ISSN 0960-1686. doi: http://dx.doi.org/10.1016/0960-1686(90)90331-G. URL http://www.sciencedirect.com/science/article/pii/096016869090331G.

A. Russell and R. Dennis. NARSTO critical review of photochemical models and modeling . Atmospheric Environment, 34(12-14):2283 - 2324, 2000. ISSN 1352-2310. doi: http://dx. doi.org/10.1016/S1352-2310(99)00468-9. URL http://www.sciencedirect.com/science/ article/pii/S1352231099004689.

U.S.EPA. Guideline for Regulatory Application of the Urban Airshed Model. Technical report, EPA-450/4-91-013. U.S. Environmental Protection Agency, Office of Air Quality Planning and Standards, Research Triangle Park, NC., 1991. 